\title{
Increasing Neonatal Healthcare Productivity using Clinical Reasoning Coaching Tools
}

Mossad AbdelHak Shaban Mohamed ${ }^{1}$, Taher Halawa ${ }^{2}$, Taufiq Hidayat ${ }^{1}$, Asrar Abu Bakar $^{1}$, Azamin Anuar ${ }^{1}$, Maurie Visvakumar ${ }^{2}$

${ }^{1}$ Department of Paediatrics, Kulliyyah of Medicine, International Islamic University, Malaysia

2Department of Paediatrics, Kulliyyah of Medicine, King Abdelaziz university, Jeddah, SÁ

Introduction: Productivity in medical field has inherent value in terms of improving our lives, which can expand our economies. Productivity in medicine has many aspects including improving clinical diagnostic skills, safety, and quality and quantity care. This study will assess whether early exposure to structured clinical reasoning coaching tools would improve their clinical decision making and productivity. Research question: Does clinical reasoning coaching tools Increase neonatal healthcare productivity? Materials and method: Medical practitioners recently joined neonatal units will participate over 2 years in an innovative series of clinical reasoning coaching sessions blended with virtual patients. Practitioners will be exposed to many teaching methods during the neonatal training that includes lectures, bedside teaching sessions and small group discussions beside website continuous contact for learning and chairing skills. Teaching series scope should cover resuscitating sick neonates, handling ventilators either conventional or high frequency, practicing common neonatal procedures, dealing with common neonatal scenarios, infection control policy and, effective communication skills The evaluation sessions will be introduced at the beginning of their training, during the course and at the end of the clerkship to assess their improving productivity, using diagnostic thinking inventory(DTI). Selection of the medical practitioners will be based on either on stratified random sampling or cohort control depending on the funding and logistic. All items will be analysed advanced statistical analysis methods. Results: The coaching tool may yield dramatic impact, allowing the innovators to be more productive. Suggesting widely utilize it for nurses, undergraduate and postgraduate medical Subspecialty. Conclusion: The research hypothesis is assuming that DTI scores and productivity will be higher after the coaching sessions as rated by the candidate's performance. 